AVANT, Vol. X, No. 2/2019

ISSN: 2082-6710 avant.edu.pl/en

DOI: 10.26913/avant.2019.02.27

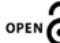

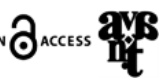

\title{
Aspect seeing and mathematical representations
}

\author{
Valeria Giardino \\ CNRS - Archives Henri-Poincaré \\ Philosophie et Recherches sur les Sciences et les Technologies \\ valeria.giardino@univ-lorraine.fr \\ Jan Wöpking \\ Independent Researcher
}

Received 10 July 2018; accepted 20 November 2018; published 31 December $2019^{1}$

\begin{abstract}
In the present article the productive role of visual representations in mathematics is explained by referring to "aspect seeing". First, the notion of aspect seeing is introduced, as proposed by Wittgenstein in his works. Second, the pragmatic dimension of aspect seeing is discussed. Third, three key examples of aspect seeing in mathematics are presented with the aim of showing the relevance of aspect shifting in important areas of mathematics by looking at both its pragmatic dimension and its relationship with conceptual mastery. Finally, some conclusions are given about aspect seeing as a human phenomenon.
\end{abstract}

Keywords: representation; mathematics; aspect seeing; visual; perception; Wittgenstein

\section{Introduction}

\subsection{Mathematical representations as "arenas of potentiality"}

In their book on the culture of diagrams, Bender and Marrinan (2010, p. 23) argue that the figures in the assemblage-like plates in Diderot and D'Alembert's Encyclopédie serve as an "arena of potentiality that fosters connections without fixing them or foreclosing thought experiments". In the present article, we will borrow this claim and extend it beyond their case study by maintaining that one of the most fascinating and epistemically relevant features of visual representations (diagrams as well as formulas) in mathematics is precisely that they prompt their users to see a given content in many and possibly radically different ways. Conceiving a mathematical representation as an "arena of potentiality" might sound odd if one

\footnotetext{
${ }^{1}$ updated version: 9 January 2020
} 
accepts the received view, according to which mathematics is the paradigm of a formal discipline that is strictly governed by syntactic rules which fix the content of a representation once for all. If mathematics were as the received view prescribes, then there would be no place for different interpretations of the very same representation. However, we will argue here that there are in fact cases in mathematics where representations may display content in newsometimes even surprising —ways.

Similar claims have recently attracted some attention in the philosophy of mathematics: Shin (2002, pp. 91-97) introduced the concept of "multiple readings" into the analysis of Peircean logical graphs; Giaquinto (2007), Grosholz (2007), Macbeth (2010) and Coliva (2012) suggested that (at least certain kinds of) mathematical proofs are based on the ability to have different views of the same visual object. Some of these authors (Macbeth, Coliva) explicitly referred to Wittgenstein's notion of aspect seeing; in this article we will attempt to go even further and discuss Wittgenstein's ideas in relation to the use of heterogeneous representations in mathematics in general, beyond the case of Euclidean geometry. We agree with authors such as Floyd that although Wittgenstein's remarks on mathematics do not provide a workedout or systematic philosophy, they "point, suggestively, toward a range of specific ways in which talk of mathematical experience and practice, even of intuition, might make sense" (Floyd 2012, p. 230). In our view, a better understanding of the notion of aspect seeing would lead to a broader and more complex understanding of the productive role of visual representations in mathematics. In the remainder of this section, we will retrace Wittgenstein's discussion of aspect seeing and then relate it to the emergence of multiple interpretations, in particular in the case of mathematics. ${ }^{2}$

\subsection{Aspect seeing: there is more than ducks and rabbits}

Consider the picture of the double cross, as presented by Wittgenstein in Part II of Philosophical Investigations (Fig.1). This picture brings attention to a radical tension, since-as Wittgenstein explains - even a pre-linguistic child would immediately react to it as an adult would "by pointing alternately to an isolated white and an isolated black cross" (Wittgenstein, PI, p. 207).

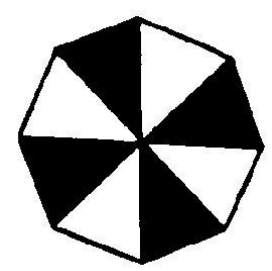

Figure 1. The double cross.

\footnotetext{
${ }^{2}$ We will limit our discussion to cases of aspect seeing because they are relevant to mathematics, despite the fact that Wittgenstein talks about aspect perception in a more general sense (for example, by including cases of aspect hearing).
} 
Phenomena such as the double cross are widely investigated in psychology, in particular in relation to a component of perceptual organization known as figure-ground organization. The Danish psychologist Rubin (1921) studied percepts such as the double cross. ${ }^{3}$ By looking at the differences between the subjective experiences of these percepts, Rubin distinguished two regions in them: figure and ground. The figure seems to appear closer to the observer and has the shape imported by the contour, while the ground appears farther away and extends behind the contour. The relation between attention and figure-ground organization is complex. As Palmer (1999, p. 284) explains, "the more likely dependency is that attention is drawn to the figure rather than that attention determines the figure". In other words, attention tends to be driven by figure-ground organization rather than the other way around. Moreover, there is a sense in which the factors that influence figure-ground organization are determined by a distinction between the objects and spaces between them; it seems plausible that figure-ground processing depends on whether the shape of a region corresponds to that of a known, meaningful object. Further experiments have shown that figure-ground organization is indeed affected by past experience - in some cases by the knowledge of specific object shapes (e.g. Peterson, Harvey \& Weidenbacher, 1991; Peterson \& Gibson, 1993).

Past experience is also relevant in other less simple cases, such as in Wittgenstein's wellknown example of the duck-rabbit picture. The picture depicted in Fig.2 is indeed a prototypical 'picture puzzle': in seeing it, one sees one picture but has two different visual experiences. ${ }^{4}$ In fact, we can see it as a picture of a duck $O R$ as a picture of a rabbit without being able to hold these two visual impressions simultaneously (Wittgenstein PI, p. 194).

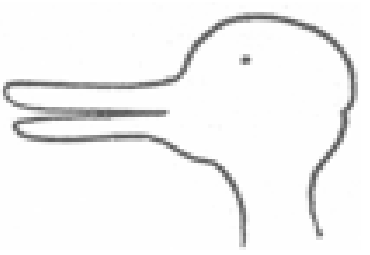

Figure 2. The duck-rabbit.

In psychological terms, these figures are "potent demonstrations of the interpretative nature of vision" (Palmer, 1991, p. 9). First, the interpretations are mutually exclusive, and this is consistent with the idea that perception involves the construction of an interpretative model since only one such model can fit the sensory data at one time. Second, they are multistable as the two possible interpretations alternate back and forth as one keeps looking at them; this suggests that the two models compete with each other.

\footnotetext{
${ }^{3}$ Other examples are the Necker cube or the vase whose contours are two symmetrical profiles of two faces. Wittgenstein himself refers to the Necker Cube (Cf. TLP 5.5423, PI, p. 193).

${ }^{4}$ Wittgenstein took this picture from a game for children designed by the experimental psychologist Jastrow (1900, Fig. 19).
} 
The double cross and the duck-rabbit picture are rather specific examples of aspect seeing. In fact, both of them involve a primarily visual form of perception that is holistic since it concerns the picture as a whole and not the way the various parts are related within the picture; most importantly, as the vast majority of participants in common language games would easily experience, the perception is exclusively binary: one can see either one aspect or the other but the two impressions cannot be held simultaneously. Nonetheless, pictures such as these have been cleverly constructed to be ambiguous, therefore our perception of them is not typical of normal processing. Moreover, ambiguity generally does not arise either in the real world or in most pictures: "rather than having constantly shifting interpretations, we usually see a stable and organised world" (Bruce et al., 2003, p. 122). Wittgenstein makes use of multistable pictures as convenient illustrations of the relevance of aspect perception in general; however, we argue that such pictures are not to be taken as the prototype of all forms of aspect seeing because instability is neither the most characteristic nor the most interesting feature of this form of seeing. Indeed, the discussion about Wittgenstein's notion of aspect seeing can be expanded by considering other examples and by avoiding focusing only on specific and extreme cases in which the observer is forced to choose from conflicting aspects. The literature has in most cases considered this specific form as the kind of aspect perception this philosopher was most interested in. ${ }^{5}$ Moreover, the experience of perceiving an aspect has been considered as mainly visual and conceptual in that one has to acknowledge one aspect or the other as meaningful. Contrary to this narrow view, it is a fact that throughout his works Wittgenstein presents a vast set of various examples of aspect perception. One of our objectives in this article will be to show the pragmatic dimension of aspect seeing: in order to explain the productivity of mathematical representations, it is necessary to consider not only a shift of interpretation but also its consequences; the choice of one interpretation among others leads the user to modify and transform the representations accordingly.

\subsection{Ambiguity and aspect-shifting in mathematics}

As Floyd (2010, p. 314) pointed out:

some of the most vivid, natural, and compelling uses of the idea of seeing aspects, interpreting one system in another, or being struck by a new aspect of a diagram, word, or sentence-as well as the earliest, most frequent, and systematic appearances of these themes in his philosophy — occur in Wittgenstein's discussions of mathematics and logic.

This attention to the role of mathematical representations in prompting aspect seeing is nonetheless not common to the standard philosophical approaches to mathematics. However, a number of scholars have focused on the dynamic aspects of mathematics and on the functioning of the various tools that are used in practice. Some of these scholars have focused in particular on features of mathematical reasoning for which aspect seeing is relevant. For example, Grosholz considered the use of representations in the historical context of problem-solving and claimed that "an epistemology that works properly for mathematics" will have to take into account "the pragmatic as well as the syntactic and semantic features of representation in

\footnotetext{
${ }^{5}$ Notable exceptions are among others the works of Floyd (2010, 2012), and Mühlhölzer (2002, 2010).
} 
mathematics" (Grosholz, 2007, p. 23). This change of perspective involves the consideration of all kinds of mathematical representations - all their "forms" - no matter whether they are diagrams or formulas: different ways of conveying mathematical information are available and each of them has its own powers and limits that should be pondered when working with them; for example, depending on the problem to solve in the context of a theory or in a particular historical moment, or for reasons of convenience, one form can be favored over another. As Grosholz explains, "different modes of representation in mathematics bring out different aspects of the items they aim to explain and precipitate with differing degrees of success and accuracy" (Grosholz, 2007, p. 258). In this view, controlled and highly structured ambiguity plays a central role in mathematical reasoning. Recall Bender and Marrinan's terminology: mathematical representation constitutes an "arena of potentiality".

Giaquinto (2007) also pointed out several dynamic features of the practice of mathematics. In the final chapter of his book, he claims that if mathematics is considered as a whole, none of the common binary distinctions that have been proposed so far between algebraic thinking and geometric thinking are of help: the philosopher's aim should be to move towards a much more discriminatory taxonomy; aspect-shifting is precisely one form of mathematical thinking that eludes standard distinctions. Take, for example, one of the many visual proofs that can be given for Pythagoras's Theorem, shown in Fig.3.

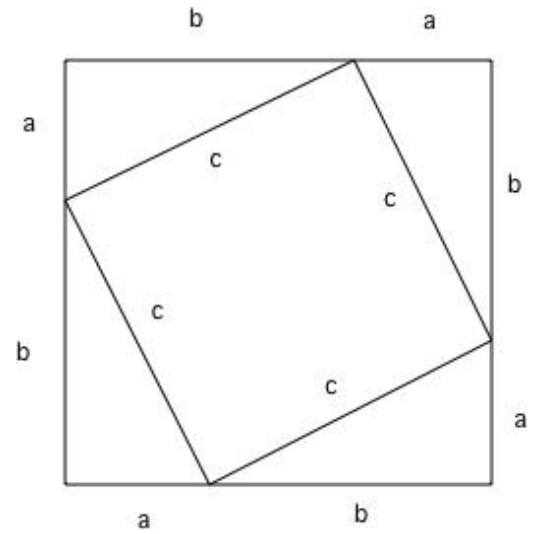

Figure 3. One visual proof (among many) of the Pythagorean theorem.

As Giaquinto (2007, pp. 240-241) explains, it is possible to look at the figure-which has labels in it - and see that the area of the larger square is equal to the area of the smaller square plus the area of the four right-angled triangles. Therefore, by reasoning "geometrically" and shifting between aspects, one recognizes that the area of the square is

both $(\mathrm{a}+\mathrm{b})^{2}$ and $2 \mathrm{ab}+\mathrm{c}^{2}$.

From here, it is easy to proceed "algebraically" as follows:

$a^{2}+2 a b+b^{2}=2 a b+c^{2}$

$a^{2}+b^{2}=c^{2}$. 
At this point, by looking back at the figure, one realizes - again "geometrically" - that the smaller square is also the square of the hypotenuse of the right-angled triangle. Finally, one concludes from the formula that the area of the square of the hypotenuse is equal to the sum of the squares of its other two sides. Is this argument primarily algebraic or geometric? Neither of these two categories seems fully appropriate to capture it.

In the remainder of the article, we will investigate the role of aspect seeing in mathematical representation. First, we will argue - in line with Grosholz - that it is necessary to shed light on the pragmatic dimension of aspect perception, as Wittgenstein also suggests (Section 2). Second, we will present three key examples of aspect seeing in mathematics (Section 3); in doing so, we will follow Wittgenstein's suggestion and resist the use of a "one-sided diet" of examples (Wittgenstein PI, §593). Finally, we will draw some conclusions on the relevance of aspect seeing for understanding the practice of mathematics (Section 4).

\section{The pragmatic dimension of aspect seeing}

\subsection{Beyond simple seeing}

In Philosophical Investigations, Wittgenstein states a fundamental difference between simple seeing - 'I see this': I see a wall, I see a man, I see lines—and seeing-as or seeing-in. ${ }^{6}$ The latter can be identified with aspect seeing and comes in two main forms: one can either (i) see a likeness between two materially different things, for example a likeness between two faces, or (ii) see two different objects in the same material thing, as in the already discussed multistable percepts. This claim is also true of diagrams and of other forms of mathematical representations. Following Wittgenstein, we argue that aspect seeing consists not only in seeing different contents in the same diagram but also in seeing the same content in different diagrams (or other kinds of representations). This is a crucial remark because many discussions of aspect seeing have been limited to the first case; we suggest instead that aspect seeing should instead be regarded as a family resemblance concept comprising quite diverse cases. However, Wittgenstein himself identifies one common feature: all cases of aspect seeing are "the experience of a comparison" (Wittgenstein, RPP I, §317). On a general level, this comparison is usually displayed either as seeing one thing as different objects or as seeing different things as the same object.

It is important to note that seeing something as or in something else is not always a primarily visual experience, as the double cross example might wrongly suggest. Wittgenstein explicitly calls our attention to the fact that only some cases of aspect seeing exclusively involve vision: "I should like to say: there are aspects which are mainly determined by thoughts and associations, and others that are 'purely optical', they make their appearance and alter automatically, almost like after-images” (Wittgenstein, RPP I, §970; cf. §989). In general, as we have already

\footnotetext{
${ }^{6}$ Consider the opening paragraph of section xi in Part II of the Philosophical Investigations (Wittgenstein, PI, p. 193): "Two uses of the word "see". The one: "What do you see there?"- "I see this" (and then a description, a drawing, a copy). The other: "I see a likeness between these two faces"- let the man I tell this to be seeing the faces as clearly as I do myself. The importance of this is the difference of category between the two 'objects' of sight".
} 
discussed, aspect seeing presupposes some level of conceptual mastery. For example, the familiarity with something that we recognize as an object - for which we might have a concept - can have an influence on our figure-ground organization. As Schroeder sums up, "aspect perception is concept-laden" (Schroeder, 2010, p. 357). Of course, when it comes to mathematics, most cases of aspect seeing are only partially visual and require much more sophisticated conceptual abilities.

Consider again the duck-rabbit picture and focus on the experience of seeing a change in the aspect. What happens when the aspect changes and one sees as the picture of a rabbit something that one formerly saw as a picture of a duck? Does this constitute some special perceptual experience? Yes, it does, but not as an exclusively visual experience: when the aspect changes, one understands that the figure can be treated also according to the concept 'rabbit', and what is experienced is the possibility of a new conceptualization. Seeing an aspect means experiencing a conceptual relation; in Wittgenstein's words, "what I perceive in the dawning of an aspect is not a property of the object, but an internal relation between it and other objects" (Wittgenstein, PI, p. 212). However, this experience is elicited by considering the physical features of the representation. The question is: does the experience of a conceptual relation have (pragmatic) consequences for the way we react to the picture?

\subsection{Seeing aspects and (re)acting accordingly}

Up to now, we have argued that aspect seeing differs from simple seeing, and that seeing a new aspect implies the experience of a new conceptual relation. Now, we want to claim that in order to give a thorough account of aspect seeing, it is crucial to remark that seeing an aspect usually also has pragmatic consequences: depending on what one sees in a representation, one reacts differently to it and chooses to operate on and with it accordingly. To go back to the duck-rabbit picture, seeing the picture as a picture of a duck prompts us to act in ways that are different from those prompted by seeing the same picture as a picture of a rabbit. Seeing something as something thus primarily consists in treating the object as something. Aspect perception has an essential pragmatic dimension. To clarify our claim, we will elaborate on the following example, given in Part II of Philosophical Investigations.

Consider the two shapes - let us call them Shape A and Shape B-shown in Fig. 4 (Wittgenstein, PI, p. 198). Shape $A$ is a shape that may or may not be symmetrical and, most importantly, it does not look familiar to us: we cannot find any label with which to name it and it does not seem to elicit any concept in us since we are not sure of what, if anything, it alludes to. ${ }^{7}$ The case of Shape $B$ is very different: we have no difficulties in recognizing it not only as a sequence of letters but also as one that is meaningful.

\footnotetext{
${ }^{7}$ It might represent a lamp on a table, but there is no way to know for sure.
} 


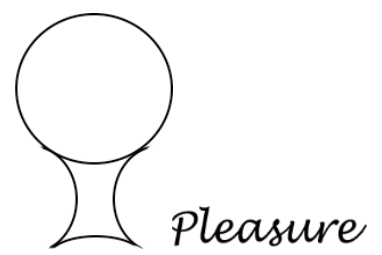

Figure 4. Shape A and Shape B respectively.

Now, imagine manipulating both shapes, for example by reflecting them vertically as in Fig. $5^{8}$.

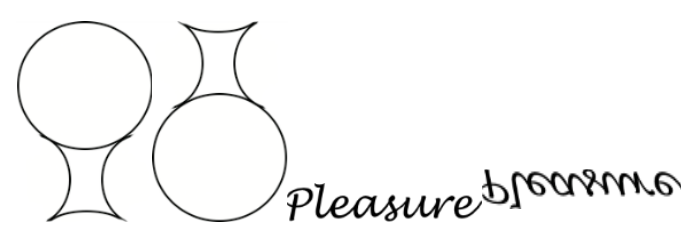

Figure 5. Shape A and Shape B and their reflected versions

The difference between the visual impressions of Shape $A$ and its reflected version is not nearly as big as that between Shape $B$ and its reflected version. Why is that? In line with what Lycan (1971) suggests, our reply to this question is that in the case of Shape A and its reflected version, both figures are equally easy to represent, i.e. to reproduce, while in the case of Shape B and its reflected version, one figure is far more readily reproduced than the other because we have been trained and have learnt how to do it.

Aspect perception has in fact a pragmatic dimension: how we perceive aspects in most shapes depends on how (and whether) we use them (and vice versa). For example, an observer mastering the European writing system is ready to recognize something - a word-in Shape B, while Shape A and its reversed version do not elicit any particular context of use. Conversely, to have pragmatic consequences of our behavior-for example, in the actions that we may perform in order to meaningfully reproduce or manipulate it - is a criterion for an experience to be accounted for as the perception of a particular aspect. This can also be defended for the duck-rabbit picture because, as Lycan (1971, p. 232, emphasis added) claims,

It is a matter of fact that, in general, when some person S, looking at the duck- rabbit, is asked to copy what he sees, or to draw the same animal in another position, or to provide a

\footnotetext{
${ }^{8}$ To be true to Wittgenstein, in the example he gives, Shape $A$ is reflected vertically and Shape B horizontally. We chose to reverse both vertically to avoid any incongruence between the two cases. However, this change does not affect Wittgenstein's argument.
} 
context for it, he responds in a consistent way; i.e., he does not fulfill conflicting criteria.

He does not, for example, draw an animal with long ears and web feet.

However, not just any action will do. Wittgenstein discusses at one point the case of a picture depicting a random curve that divides the area of a rectangle in two (Wittgenstein, RFM, § I70). In principle, this picture could be regarded as a proof that a rectangle can be divided into two parts by a such-and-such a curve. However, as Wittgenstein hastens to points out, such a picture would not be interesting to us and is pointless as a proof since it does not enable us to take any action whatsoever to make some aspect emerge in order to provide a proof. Following Baz (2000), to notice an aspect must also mean to find something of value in a representation; this value is often expressed by wonder, astonishment or surprise: "I see something that isn't there at all, that does not reside in the figure, so that it may surprise me that I can see it" (Wittgenstein, RPP I, §1028). There are different ways in which an aspect can be of value for an agent. One way in which an aspect can have value is the extent to which something can be done thanks to its acknowledgement, in other words, when it has practical value (Mühlhölzer, 2010, pp. 326-328). In the following section we will see how the pragmatic dimension of aspect seeing can be modulated in relation to different mathematical representations.

\section{Seeing aspects in mathematics: Case Studies}

In this section we will refer to three case studies to discuss in more detail the features of aspect seeing in mathematics. We have three main goals: (i) to show the relevance of aspect seeing for important areas of actual mathematics; (ii) to make a case for the heterogeneity and pervasiveness of the various kinds of aspect seeing in mathematics; (iii) to present the pragmatic dimension of aspect seeing in action while at same time showing its intimate interrelatedness with conceptual mastery in the relevant area.

Before introducing the case studies, we have to emphasize that in Wittgenstein's discussion, aspect seeing manifests itself in different contexts: some of these can be explicitly found in his writings, while some are only hinted at by him and others can be derived by interpreting his remarks. The mathematical examples discussed by Wittgenstein and by the literature on his thought are very diverse, both from the point of view of the involved area of mathematics and of the function played by aspect seeing in mathematical arguments. The examples we will give here are not taken from Wittgenstein's writings, but nonetheless we argue that they serve as a good illustration of his ideas. Most importantly, they allow us to discuss the relevant implications of aspect seeing for the philosophy of mathematics by showing the different forms that aspect seeing may take in the specific case of mathematical reasoning: in the first example, different configurations are seen in the same geometrical diagram; in the second example, different aspects of the same mathematical structure are presented in one diagram; in the third and final example, the same representation leads to different implicit inferences. 


\subsection{First case study: Euclidean geometry}

At one point in his discussion of aspect seeing in Philosophical Investigations, Wittgenstein himself singles out a specific class of aspect seeing when he remarks:

One kind of aspect might be called 'aspects of organization'. When the aspect changes parts of the picture go together which before did not. In the triangle I can see now this as apex, that as base - now this as apex, that as base. - Clearly the words "Now I am seeing this as the apex" cannot so far mean anything to a learner who has only just met the concepts of apex, base, and so on. - But I do not mean this as an empirical proposition. "Now he's seeing it like this", "now like that" would only be said of someone capable of making certain applications of the figure quite freely. The substratum of this experience is the mastery of a technique. (PI, p. 208)

There is no better example than Euclidean geometry to show what Wittgenstein had in mind here. Take Euclid's Elements, Book I, Proposition 9: to bisect a given rectilinear angle. As for Euclidean proofs in general, this proof can also be divided into two: the construction phase in which a diagram gets constructed, and then the inference phase in which it is shown that various inferences can be read off from the constructed diagram, and this would lead eventually to the proof of the proposition. ${ }^{9}$ These inferences are licensed by definitions and axioms and previously proved propositions.

The construction phase is as follows:

"To bisect a given rectilinear angle.

Let the angle $\mathrm{BAC}$ be the given rectilinear angle.

Thus, it is required to bisect it.

Let a point $\mathrm{D}$ be taken at random on $\mathrm{AB}$;

let $\mathrm{AE}$ be cut off from $\mathrm{AC}$ equal to $\mathrm{AD}$; [I.3]

let DE be joined, and on DE let the equilateral triangle DEF be constructed; [I.1]

let AF be joined". (Heath 1956, p. 264). ${ }^{10}$

The result of this construction phase is Fig. 6 .

\footnotetext{
${ }^{9}$ Actually, Euclidean proofs are divided into five phases, but for our present purposes it is sufficient to distinguish between construction and inference phase. For recent discussions of the five phases in more detail see Shin (2012) and Catton and Montelle (2012).

${ }^{10}$ The annotations in square brackets indicate previous propositions in virtue of which the before mentioned construction can be carried out.
} 


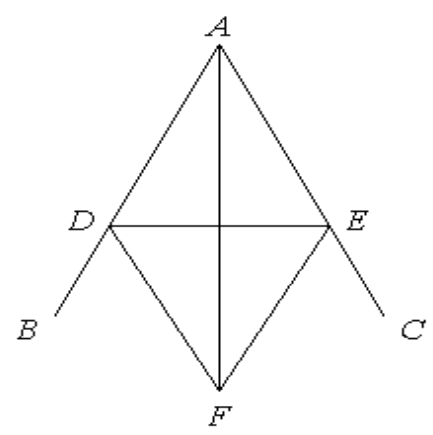

Figure 6. Euclid I.9.

Then starts the inference phase:

"I say that the angle BAC has been bisected by the straight line, AF.

For since $\mathrm{AD}$ is equal to $\mathrm{AE}$,

and $\mathrm{AF}$ is common,

the two sides $\mathrm{DA}, \mathrm{AF}$ are equal to the two sides $\mathrm{EA}, \mathrm{AF}$ respectively.

And the base DF is equal to the base EF;

Therefore, the angle DAF is equal to the angle EAF [I.8].

Therefore, the given rectilinear angle BAC has been bisected by the straight line AF". (Heath 1956, p. 264).

In the inference phase, Euclid tells us to pay attention to two triangles emerging from the construction. Thanks to the way the construction was carried out, we know that these are triangles of which each pair of corresponding sides has the same length. Euclid uses this similarity between the two triangles by applying something he had previously proved. In I.8 he demonstrated that "If two triangles have the two sides equal to two sides respectively, and have also the base equal to the base, they will also have the angles equal which are contained by the equal straight lines". (Heath, 1956, p. 261). As the two triangles match the premises of I.8, the conclusion of this proposition also holds: their angles are of equal size. As DAF and EAF have the same size, and as they—as we can infer directly from the diagram - make up the whole angle BAC, we have achieved the bisection of the angle.

As with most of Euclid's proofs in the Elements, the most remarkable thing to note about I.9 is that in both phases one is dealing with quite different geometric entities. In the construction phase, one is concerned with the angle BAC, the line DE, and the equilateral triangle DEF. In the inference phase, however, one does not refer to these anymore; instead, one focuses on the 
triangles DAF and EAF and proceeds by referring to "wholly new geometrical entities" (Macbeth, 2009, p. 375) within the figure, which in turn enable the user to draw various inferences. As Macbeth argues, the key to Euclidean proofs is the potential of the diagram "to be regarded in radically different ways" (2009, p. 376). This requires the ability of aspect seeing in the user. We fully agree on this point; however, we disagree with her when she characterizes this ability as a "perceptual skill" (Macbeth, 2009, p. 377): aspect seeing in mathematics is more than just a perceptual skill, since it already presupposes the mastery of the relevant mathematical practice. ${ }^{11}$

Let us now take a closer look at the interrelation between the pragmatic dimension of aspect seeing and the mastery of the practice of Euclidean geometry. First, Euclid focuses on one particular organizational aspect of the picture, to which he applies a previously proved proposition. Here, aspect seeing enables him to make epistemic progress: to see the two triangles is the epistemic crux of the proof. Once one sees the two triangles, the rest of the proof follows easily by applying elementary inference rules. The epistemic relevance of this move is of great importance for our argument since, as is evident, there would have been many other figures that one could have recognized from the construction, for example the quadrilateral ADFE. But we are not interested in seeing ADFE in the picture, because seeing this aspect in the picture would not have any practical consequence - it is pointless, just as is the case of seeing the random curve as dividing the area of the rectangle mentioned above.

This example is also useful to clarify that seeing aspects in mathematics is by no means exclusively or even primary purely visual. There are two main reasons for this, the first and most obvious of which is that one needs to know the definitions and the syntactic rules of Euclidean geometry in order to be able to see three connected lines on a sheet of paper as geometrical angles, triangles, sides and so on, with their specifically defined properties. Moreover, one also needs to be aware of the content of the previously proved propositions in order to know that ADF and EAF really conform to the premises of Proposition I.8. As a consequence, mastery of a minimal conceptual framework is a prerequisite for being able to see ADE and EAF as two triangles that match the premises of Euclid I.8. At this point, one might object that actually and literally seeing $\mathrm{AD}$ and $\mathrm{AE}$ and $\mathrm{DF}$ and $\mathrm{EF}$ as sides of equal length respectively and so on was a condition for being able to see ADF and EAF as two similar triangles in the figure (Giaquinto, 1993, pp. 88-91). ${ }^{12}$ However, this objection is based on a mistaken assumption about the role that diagrams play in this proof. As Manders (2008) showed in his seminal paper on the Euclidean Diagram, only some of the inferences of a proof may be read off from the diagram, while others are licensed by the accompanying text. Manders calls the first coexact and the latter exact features of a diagram. Exact features are, for example, the length of

\footnotetext{
${ }^{11}$ One possibility could be to accept with Catton's and Montelle's contraposition of "manual practice" versus "concept-bound performance of a seeing-as" (2012, p. 54).

12 "What is it to see a diagram (or part of it) as a square? It is not to see the diagram and, as a result of this seeing, to believe that it is square; nor is it the diagram's appearing square when we see it. As noted above, it may appear not exactly square. But it must appear, if not exactly square, at least roughly square. It must look similar to something which appears exactly square. Seeing the diagram as a square involves both seeing it and observing this similarity of appearance" (Giaquinto, 1993, p. 90).
} 
the sides, their parallelness, the size of angles, and so on; they vary greatly due to small deformations in the figure, while co-exact properties are stable even under great perturbations of the figure. Examples of such co-exact properties are part-whole or incidence relations: they can be safely inferred from the diagram without fear of endangering the generality of the proof. ${ }^{13}$ Manders' argument implies that diagrams can be drawn quite imprecisely without losing their generality and appropriateness for proofs.

Wittgenstein raises a similar point in his Remarks on the Foundations of Mathematics:

The drawing of a Euclidian proof may be inexact, in the sense that the straight lines are not straight, the segments of circles not exactly circular, etc. etc. and at the same time the drawing is still an exact proof; and from this it can be seen that this drawing does not- e.g.demonstrate that such a construction results in a polygon with five equal sides; that what it proves is a proposition of geometry, not one about the properties of paper, compass, ruler and pencil. (RFM, § II, 1)

But if this is true, then it reinforces our point that aspect seeing is an activity that is in part concept-laden. Would it be possible in principle to see the two triangles ADF and EAF as similar in the sense of I.8 even if they did not look optically similar? We could do this by referring to aspect seeing and thus taking into account the additional and equally essential information provided by the text of the construction. In sum, only by mastering the complex and subtle interplay between exact features represented by the textual component of the proof and the co-exact features represented by its diagrammatic elements is it possible to see two triangles in the figure.

\subsection{Second case study: diagonalizations}

Our second example is Cantor's pairing function, which shows that the set of rational numbers is countable, i.e. that there exists a bijection between $Q$ and $N .{ }^{14}$ The proof is given by arranging the rational numbers in a way that makes sure that (i) no element is left out and (ii) the elements can be brought into a 1-to-1 correspondence with the elements of the set of natural numbers, i.e. they can be counted (see Fig.7) ${ }^{15}$.

\footnotetext{
${ }^{13}$ For details see Manders (2008), Avigad et al. (2009), Mumma (2010); see also Sherry (2009).

${ }^{14}$ For reasons of simplicity we only deal with the positive rationals $Q+$. The argument can, of course, be extended to apply to all of $Q$ including the negative rationals $Q-$.

${ }^{15}$ The diagram is taken from Roitman (1990, p.77).
} 


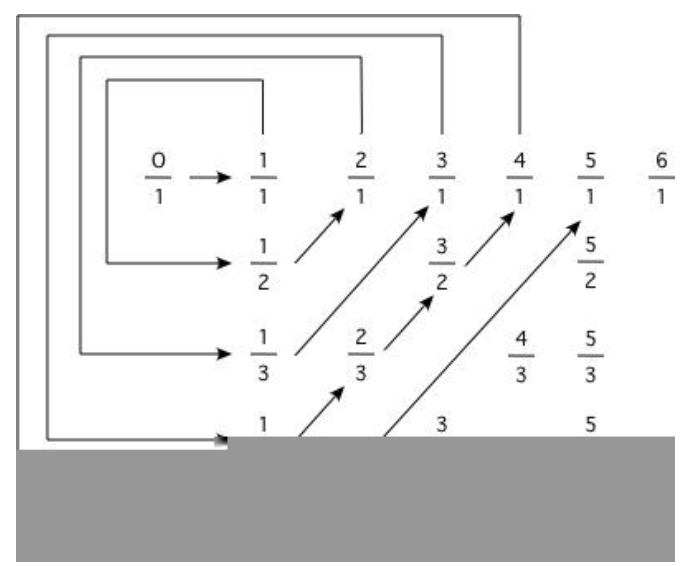

Figure 7. Cantor's pairing function.

This example involves seeing two different sets in the diagram: (i) the set of rational numbers (being represented by the fractions) and (ii) the set of natural numbers (being represented by the arrows). By linking together these two different representations, one realizes that there is a bijective mapping from the naturals onto the rationals. Following Oliveri (1997), we take this specific ordering to be an aspect of the set of rational numbers; this is one way in which we can see the rational numbers as being arranged. Such an arrangement constitutes the center of the proof; this surprises and puzzles us at first, and later — once we recognize the validity of the proof - it becomes the reason for us to keep admiring its elegance. As Mühlhölzer claims, for Wittgenstein surprise in mathematics "does not arise out of the discovery of new, astonishing facts but from a new representation, or a new arrangement, of facts; of facts which per se may be quite banal" (Mühlhölzer 2002, p. 310) ${ }^{16}$.

In Euclidean proofs, an aspect-seeing rationale is built in from the beginning; this is different in the case of Cantor's argument, where aspect seeing consists in a creative reordering of the rationals. As in the case of Euclid, this kind of aspect seeing makes use of the two-dimensionality of the page: it is a nice example of an "intelligent use of space" (Kirsh 1995), namely an external representation of a problem whose form facilitates or enables a solution. Differently from the case of Euclid, no auxiliary lines are introduced here (the arrows only serve as indexes); however, one can argue that the creative use of the plane plays a similar role since it is the element that brings out the epistemically helpful aspects.

In Cantor's example, we find a new aspect of the rationals; however, it is of course equally possible to rearrange the naturals in a new way. Think of the famous Gaussian argument for the sum of the first $n$ natural numbers, call it $\mathrm{S}(n)$. The numbers 1 to $\mathrm{n}$ can be represented in the following way:

\footnotetext{
${ }^{16}$ See also Floyd (2012) on surprise in Wittgenstein's philosophy of mathematics
} 


$\begin{array}{lllllll}1 & 2 & 3 & \ldots & (n-2) & (n-1) n & \\ N & (n-1) & (\mathrm{n}-2) & \ldots & 3 & 2 & 1\end{array}$

Now, this new arrangement can be seen in two ways: (i) as two rows, each having $\mathrm{S}(n)$ as a sum; (ii) as $n$ columns consisting of two addends which in each case add up to $(n+1)$. Combining these two aspects, we get $2 \mathrm{~S}(n)=n(n+1)$, which leads to $\mathrm{S}(n)=n(n+1) / 2$. This technique of seeing two different aspects in the same structure and combining them to solve a mathematical problem lies at the heart of many other visual proofs.

\subsection{Third case study: formal verification}

At one point in Philosophical Grammar, Wittgenstein makes a remark about the cardinal number ' 1 ' and the rational number ' 1 ' as being subject to analogous rules while nevertheless being different signs (PG, p. 441). ${ }^{17}$ In a related fashion, Avigad (2008) discusses the case of how we understand numeric substructures.

According to a foundational perspective, natural numbers are considered as basic: integers are viewed as equivalence classes of pairs of natural numbers, rational numbers as equivalence classes of integers, real numbers as sequences of rational numbers, and finally complex numbers as pairs of real numbers. ${ }^{18}$ Nonetheless, this image contrasts with our tendency to think of natural numbers as a subset of integers, of integers as a subset of rational numbers, and so on. Of course, we can choose at any time to consider the "original copy" of the cardinal (natural) number ' 1 ' as its rational or even complex image ' 1 ', but if we do so then we have to keep track of "where individual elements 'live" each time we consider them (Avigad 2008, p. 249) in order to understand how they will behave in arguments. For example, we may apply the principle of induction to the complex copy of the natural numbers, but not to complex numbers as a whole. As Avigad points out, such cases of aspect shifting are ubiquitous and elementary in everyday mathematics, but they tend to pass completely unnoticed. In a textbook, it will never be explicitly specified which of the possible images of the numerical structures one should refer to; despite this, in reading a theorem we will be able to understand how the statements in the argument are to be interpreted (provided that we are sufficiently trained). This form of aspect seeing appears more clearly when we see it breaking down, as happens when trying to formalize a proof using formal verification systems. In fact, proof assistants, at least the ones that have been developed so far, require explicit type conversions: inferences need to be spelt out in detail each time, and this requires time and a lot of programming effort (Avigad, 2008, p. 350).

\footnotetext{
${ }^{17}$ By cardinal number, Wittgenstein seems to mean here just natural number.

${ }^{18}$ An integer $\mathrm{x}$ is the equivalence class of a natural numbers $(\mathrm{m}, \mathrm{n})$, such that $\mathrm{x}+\mathrm{n}=\mathrm{m}$. A rational number is an equivalence class of pairs [a,b], with a and $b$ integers and two pairs $[a, b]$ and $[c, d]$ equivalent iff ad=bc. And so on...
} 
Similar considerations can be made even for more ordinary cases such as the use of common programming languages like $C$. In $C$, the result of a division of two variables of data type Integer is itself of type Integer. So, the following $\mathrm{C}$ code snippet

$$
\begin{aligned}
& \text { int } \mathrm{x}=5, \mathrm{y}=4 \text {; } \\
& \text { float } \mathrm{z} ; \\
& \mathrm{z}=\mathrm{x} / \mathrm{y} ;
\end{aligned}
$$

evaluates to ' 1 ' because the positions after the decimal point are simply ignored by the compiler. In order to get the result of ' 1.25 ', one has to explicitly cast $\mathrm{x}$ and $\mathrm{y}$ to data type float

$$
\begin{aligned}
& \text { int } \mathrm{x}=5, \mathrm{y}=4 ; \\
& \text { float } \mathrm{z} ; \\
& \mathrm{z}=\text { float }(\mathrm{x}) / \text { float }(\mathrm{y}) ;
\end{aligned}
$$

When forced to manually cast switches between data types, one gets a glimpse of how much aspect seeing and implicit contextualization are done in everyday mathematical activity. As Avigad remarks,

"[t]he fact that methods of reasoning that we are barely conscious of when we read a mathematical proof requires so much effort to formalize is one of the scandals of formal verification, and a clear sign that more thought is needed as to how we understand such inferences" (2008, p. 350).

Conversely, completely formalized proofs are rather hard if not impossible to read and understand.

In this last example, the pragmatic dimension of aspect seeing is still present: without being able to easily switch between different aspects of a mathematical representation, our understanding of a lot of proofs would break down because we would not be able to spell out the details that are left implicit. However, this is only possible once successful mastery of the practice is achieved, for otherwise one would not know when to see a number as a natural, real or complex one. These are things that have to be learnt.

\section{Conclusions}

One of our aims in the article was to show that aspect seeing is a key feature of the practice of mathematics. This is easier to see in elementary mathematics-Euclidean geometry, basic arithmetic or algebra-but it is also true for more complex areas of mathematics (e.g. Floyd 2010). Aspect seeing often determines the crucial step in a proof. Moreover, being able to discriminate between the important parts of the proof on the one hand and the rather mechanical ones on the other also depends on aspect seeing. Avigad (2008, p. 328) takes "the ability to indicate 'key' or novel points in the argument and separate them from the steps that are 'straightforward"" as a criterion for what it means to understand a mathematical proof. We have seen in our examples that aspect seeing may play an epistemically productive role; this 
is in accordance with Wittgenstein's own line of thought. At one point, he admits some skepticism towards the vagueness of his own notion of aspect seeing: "The expression 'new aspect' is vague. It means that we now look at the matter differently - but the question is: what is the essential, the important manifestation of this 'looking at it differently'?” (RFM, §III-47). For him, in our interpretation, this manifestation is precisely the actual operative and practical relevance of seeing an aspect. Compare seeing an aspect with using a notation in a different way: "Where does it stop being-just a different notation? Isn't it where only the one notation and not the other can be used in such-and-such a way?" (RFM, §III-47).

Aspect seeing is a human phenomenon not only in the case of mathematics, but also in that of the pictures shown in the first part of the paper (with the possible exception of the double cross). As in the case of the number ' 1 ' belonging either to the set of natural, real or complex numbers, human agents are able to easily shift contexts and see numbers accordingly. Computers and machines in general are incapable of doing that, and to some extent we could conceive of them as a true reification of an aspect-blind person, whom Wittgenstein thinks about a lot. The enterprise of formalism can be thought of as an effort to produce that blindness in human mathematical practice. But, as Azzouni points out, formalism does not seem to have had a significant impact on the practice of mathematics. He suggests that this could change with computers becoming more powerful in deriving new mathematical results from a given set of information and argues that computer-based derivations are ultimately more reliable because they are blind to the psychological elements that play an important though often unconscious role in human proofs (Azzouni 2005). However, besides the issues of certainty and the reliability of results, there are also the questions of being able to separate the important from the unimportant steps in a proof, to explain the relevance of certain passages, to see why something (a new way of writing or seeing) matters. These would not be included in such formalized proofs, precisely because they presuppose the ability of aspect seeing that computers do not have. The result would be a mathematical practice that would differ in much more than just the way that results are derived.

\section{References}

Avigad, J. (2008). Understanding Proofs. (In P. Mancosu (Ed.), The Philosophy of Mathematical Practice (pp. 317-353), Oxford: Oxford University Press.)

Avigad et al. (2009). A Formal System for Euclid's Elements. The Review of Symbolic Logic, 2(4), 700-768.

Azzouni, J. (2005). Is There Still a Sense in which Mathematics Can Have Foundations. (In G. Sica (Ed.), Essays on the Foundations of Mathematics and Logic (pp. 9-47). Milan: Polimetrica.)

Baz, A. (2000). What's the Point of Seeing Aspects? Philosophical Investigations, 23(2), 97-121.

Bender, J. \& Marrinan, M. (2010). The Culture of Diagram. Stanford: Stanford University Press.

Bruce, V. \& Green, P., Georgeson, M. (2003). Visual Perception: physiology, psychology and Ecology (4th ed.). Hove-New York: Psychology Press.

Catton P. \& Montelle, C. (2012). To Diagram, to Demonstrate: To Do, To See, and To Judge in Greek Geometry. Philosophia Mathematica, 20(1), 25-57. 
Coliva, A. (2012). Human diagrammatic reasoning and seeing-as. Synthese, 186(1), 121-148.

Floyd, J. (2012). Das Überraschende: Wittgenstein on the Surprising in Mathematics. (In J. Ellis \& D. Guevara (Eds.), Wittgenstein and the Philosophy of Mind (pp. 225-258). Oxford: Oxford University Press.)

Floyd, J. (2010). Wittgenstein on Aspect-Perception, Logic, and Mathematics. (In W. Day \& V.J. Krebs (Eds.), Seeing Wittgenstein anew (pp.314-337). Cambridge: Cambridge University Press.)

Friedman, M. (1985). Kant's Theory of Geometry. The Philosophical Review, 94(4), 455-506.

Giaquinto, M. (1993). Diagrams. Socrates and Meno's Slave. International Journal of Philosophical Studies, 1(1), 81-97.

Giaquinto, M. (2007). Visual thinking in mathematics. Oxford: Oxford University Press.

Grosholz, E. (2007). Representation and Productive Ambiguity in Mathematics and the Sciences. Oxford-New York: Oxford University Press.

Heath, T. (1956). The Thirteen Books of Euclid's Elements-translated from the text of Heiberg. New York: Dover.

Jastrow, J. (1900). Facts and fables in Psychology. London: Macmillan.

Kirsh, D. (1995). The intelligent use of space. Artificial Intelligence, 73(1), 31-68.

Lycan, W. G. (1971). Gombrich, Wittgenstein, and the Duck Rabbit. Journal of Aesthetics and Art Criticism, 30 (2), 229-237.

Macbeth, D. (2009). Meaning, Use, and Diagrams. Etica \& Politica, Ethics \& Politics, XI(1), 369384.

Macbeth, D. (2010). Diagrammatic Reasoning in Euclid's Elements. (In B. Van Kerkhove, J. De Vuyst \& J. P. Van Bendegem (Eds.), Philosophical Perspectives on Mathematical Practice (pp. 235-267). London: College Publications.)

Manders, K. (2008). The Euclidean Diagram (1995). (In Paolo Mancosu (Ed.), The Philosophy of Mathematical Practice (pp 80-133). Oxford: Oxford University Press.)

Mühlhölzer, F. (2010), Braucht die Mathematik eine Grundlegung? Ein Kommentar des Teils III von Wittgenteins Bemerkungen über die Grundlagen der Mathematik. Frankfurt a.M.: Klostermann.

Mühlhölzer, F. (2002). Wittgenstein and Surprises in Mathematics. (In R. Haller \& K. Puhl (Eds.), Wittgenstein and the Future of Philosophy: A Reassessment after 50 Years (Proceedings of the 24th Internationational Wittgenstein-Symposium, Kirchberg am Wechsel, 2001) (pp. 306-315). Wien: öbv\&hpt.)

Mumma, J. (2010). Proofs, Pictures, and Euclid. Synthese, 175(2), 255-287.

Oliveri, G. (1997). Mathematics. A Science of Patterns. Synthese, 112(3), 379-402.

Palmer, S. (1999). Vision Science: photons to phenomenology. Cambridge, Mass.: MIT Press.

Peterson, M. A., \& Gibson, B. S. (1993). Shape recognition inputs to figure-ground organization in three-dimensional grounds. Cognitive Psychology, 25(3), 383-249. 
Peterson, M. A., Harvey, E. M., \& Weidenbacher, H. J. (1991). Shape recognition contribution to figure-ground reversal: Which route counts? Journal of Experimental Psychology: Human Perception and Performance, 17(4), 1075-1089.

Roitman, J. (1990), Introduction to modern set theory, New York: Wiley.

Rubin, E. (1921). Visuell Wahrgenommene Figuren. Kobenhaven: Glydenalske boghandel.

Schroeder, S. (2010). A Tale of two problems: Wittgenstein's discussion of aspect perception. (In J. Cottingham \& P. Hacker (Eds.), Mind, method and morality: essays in honour of Anthony Kenny (pp. 352-371). Oxford: Oxford University Press.)

Sherry, D. (1999). The Role of Diagrams in Mathematical Arguments. Foundations of science, 14(1), 59-74.

Shin, S. (2002). The Iconic Logic of Peirce's Graphs. Cambridge, Mass.: MIT Press.

Shin, S. (2012). The forgotten individual: diagrammatic reasoning in mathematics. Synthese, 186(1), 149-168.

Wittgenstein, L. (1953). Philosophical Investigations (= PI), G.E.M. Anscombe \& R. Rhees (Eds.), G.E.M. Anscombe (Trans.), Oxford: Blackwell, second edition 1958.

Wittgenstein, L. (1956). Remarks on the Foundations of Mathematics (= RFM), G. H. v. Wright, R. Rhees \& G.E.M. Anscombe (Eds.), G.E.M. Anscombe (Trans.). Oxford: Blackwell, revised edition 1978.

Wittgenstein, L. (1961). Tractatus Logico-Philosophicus (= TLP). D.F. Pears \& B.F. McGuinness (Trans.), New York: Humanities Press.

Wittgenstein, L. (1974). Philosophical Grammar (= PG). R. Rhees (Ed.), A. Kenny (Trans.). Oxford: Blackwell.

Wittgenstein, L. (1980). Remarks on the Philosophy of Psychology, vol. 1 (= RPP I). G.E.M. Anscombe \& G.H. v. Wright (Eds.), G.E.M. Anscombe (Trans.). Oxford: Blackwell.

The editorial and publishing process of this publication has been financed by the research grant "What is Thinking with Images?", SONATA 10, granted by the National Science Centre, Poland, on the basis of the decision No. 2015/19/D/HS1/02426. 